\title{
LETTERS
}

\section{Beyond implementation research for improving maternal, newborn and child health globally}

Sharma and colleagues highlight the uneven progress in achieving improvements in maternal, newborn and child survival in low-income countries, emphasizing that "gaps in coverage of essential interventions are related to marginalization of some groups, social inequities and geo-

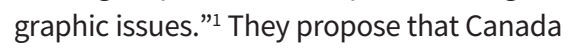
should focus on addressing this gap through implementation research. Sadly, this approach perpetuates the practices by donor companies of setting change agendas for developing countries: the "comefrom-away, top-down, we-know-whatis-needed" approach. Many developing countries are not in a position to say no, because any new resources are good, no matter what strings or directions.

Unfortunately, the proposed top-down, scale-up model is doomed to failure at the community level. Experience has shown that once donor funds disappear, programs begin to fade away if they are not locally owned and sustainable with local resources. For long-term impact in each and every community, solutions must be locally owned and address local health problems that the community sees as important. The solutions need to be evidence-based but also fit the local culture, context and resources. Come-from-away, top-down interventions rarely do this.

The authors are correct if one starts with the premise that implementation research could help, and there will be many willing to agree, but we would challenge that Canada could be more helpful if the focus is on low-income countries building their own community, district and regional capacity for finding local sustainable solutions that fit. Canada can support this through thoughtful local partnerships, but it cannot be the driver that must be done by communities. MicroResearch is one example of local communities learning how to find their own evidence-based solutions that fit their community. ${ }^{2}$

\section{Noni E. MacDonald MD MSc}

Professor, Department of Pediatrics, Dalhousie University, and IWK Health Centre, Halifax, NS

\section{Robert Bortolussi MD}

Emeritus Professor of Pediatrics, Dalhousie University, and IWK Health

Centre, Halifax, NS

\section{Jerome Kabakyenga MC PhD}

Director, Maternal Newborn Child Health Institute, Mbarara University of Science and Technology, Mbarara, Uganda

\section{John Frank MD MSc}

Chair, Public Health Research and Policy, University of Edinburgh, Edinburgh, Scotland, UK

Cite as: CMAJ 2017 May 23;189:E729. doi: $10.1503 / \mathrm{cmaj} .732980$

\section{References}

1. Sharma R, Scott H, Bhutta ZA. Enhancing implementation research within Canada's investments in the health of women and children globally. CMAJ 2017;189:E332-3.

2. MacDonald NE, Borotolussi R, Pemba S, et al. Supporting research leadership in Africa. Lancet Glob Health 2016;4:e362.

\section{Competing interests: None declared.}

All of the authors are either Co-Directors or Research Advisory Board Members of MicroResearch, an initiative to help develop local research capacity in Eastern Africa (www. microresearch.ca). 